\title{
MicroRNA-125 modulates radioresistance through targeting p21 in cervical cancer
}

\author{
ABRAHAM PEDROZA-TORRES ${ }^{1,2}$, ALMA D. CAMPOS-PARRA ${ }^{1}$, OLIVER MILLAN-CATALAN ${ }^{1}$, \\ YAHIR A. LOISSELL-BALTAZAR ${ }^{1}$, HORACIO ZAMUDIO-MEZA ${ }^{1,2}$, DAVID CANTÚ DE LEÓN ${ }^{3}$, \\ GONZALO MONTALVO-ESQUIVEL ${ }^{3}$, DAVID ISLA-ORTIZ ${ }^{3}$, LUIS A. HERRERA ${ }^{4}$, ÓSCAR ÁNGELES-ZARAGOZA ${ }^{5}$, \\ GUADALUPE ROBELO-ROMERO ${ }^{5}$, ÁNGEL HERRERA-GÓMEZ ${ }^{6}$ and CARLOS PÉREZ-PLASENCIA ${ }^{1,7}$ \\ ${ }^{1}$ Cancer Genomics Group, National Cancer Institute of Mexico, México City 14080; ${ }^{2}$ Cátedra CONACyT, \\ Insurgentes Sur 1582, Col. Crédito Constructor, Benito Juárez, México City 03940; ${ }^{3}$ Gynecology Functional Department, \\ ${ }^{4}$ Cancer Research Unit, ${ }^{5}$ Radiotherapy Department, and ${ }^{6}$ Deputy Director, National Cancer Institute of Mexico, \\ México City 14080; ${ }^{7}$ National Autonomous University of México, Cancer Genomics Group, \\ FES-Iztacala, UBIMED, Tlalnepantla, Estado de México 54090, Mexico
}

Received July 4, 2017; Accepted January 10, 2018

DOI: 10.3892/or.2018.6219

\begin{abstract}
Cervical cancer (CC) is one of the most common cancers diagnosed in women worldwide, and it is estimated that $~ 500,000$ new patients are diagnosed with cervical cancer annually and that 270,000 deaths occur each year. Patients with cervical cancer are treated with different radiotherapy schedules, either alone or with adjuvant chemotherapy. Unfortunately, nearly $50 \%$ of all patients with cervical cancer do not respond to standard treatment due to tumor radioresistance. In this scenario, several microRNAs (miRNAs) have been associated with the acquisition of the radioresistance phenotype. The aim of the present study was to evaluate the possible role of miR-125a in the acquisition of radioresistance in cervical cancer. The expression of miR-125a was assessed by means of RT-qPCR in 30 cervical cancer samples from patients receiving standard treatment and 3 induced radioresistant cervical cancer cell lines. In addition, we employed miR-125a mimics and inhibitors to evaluate its function in the induction of radioresistance. We showed that miR-125a was downregulated in patients with cervical cancer who did not respond to standard treatment. Concordantly, radioresistant $\mathrm{SiHa}$, CaSki and HeLa cell lines had low levels of miR-125a with respect to the sensitive cell lines. Finally, we demonstrated that overexpression of miR-125a sensitized cervical cancer cells to radiation therapy through the downregulation of CDKN1A. Our data corroborate previously published studies in which it was demonstrated that
\end{abstract}

Correspondence to: $\mathrm{Dr}$ Carlos Pérez-Plasencia, National Autonomous University of México, Cancer Genomics Group, FES-Iztacala, UBIMED, Av De Los Barrios 1, Tlalnepantla, Estado de México 54090, Mexico

E-mail: carlos.pplas@gmail.com

Key words: radioresistance, miR-125a, cervical cancer, $\mathrm{p} 21$ expression
miRNAs could play a role in the regulation of the process of radioresistance. Additionally, we showed that overexpression of miR-125a could be used as a radioresistance biomarker in patients with cervical cancer.

\section{Introduction}

Cervical cancer (CC) remains one of the most common cancers diagnosed worldwide, and is the third leading cause of cancer-related deaths in women. Approximately 527,600 new patients were diagnosed with CC and 265,700 deaths occurred in 2012 (1). Standard treatment depends on the clinical stage. However, radiotherapy either alone or with adjuvant chemotherapy remains the standard treatment. Indeed, radiation therapy is utilized for over $60 \%$ of patients with CC and is the first treatment choice in $\sim 52 \%$ of all patients with cancer (2).

Unfortunately, nearly $50 \%$ of all patients with CC do not respond to standard treatment due to acquired radioresistance, which is considered the main cause of related deaths associated with treatment failure in patients with CC (3). Thus, radioresistance could be defined as the ability of tumor cells to survive and repair the molecular damage caused by ionizing radiation and its effectors such as free radicals.

MicroRNAs or miRNAs are a group of endogenous, small non-coding RNAs, which are 21-25 nucleotides in length. miRNAs play a critical role in post-transcriptional gene regulation by degrading or preventing the translation of their target messenger RNA (mRNA). Recently, miRNAs have been called 'the master regulators' of gene expression, due to the fact that they have been implicated in a wide variety of cellular processes. To date, few miRNA expression profiles have been related to the radioresistance of patients with $\mathrm{CC}$ and CC-derived cell lines; for instance, miR-630, miR-1246, miR-1290, miR-3138, miR-31-3p and miR-3676 were found upregulated, whereas miR-1271, miR-15b*, miR-19b-1*, miR-378*, miR-95, miR-100-5p, miR-200a-5p, miR-320, and miR-342 were found downregulated $(4,5)$. Recently, we 
showed that patients with locally advanced CC who do not respond to conventional treatment have a specific miRNA signature, with miR-125 highlighted in that latter signature (6). In the present study, we hypothesized the importance of miR-125a as a potential key regulator for the treatment response of $\mathrm{CC}$ patients.

The miR-125 family consists of 3 homologous members: miR-125a; miR-125b-1 and miR-125b-2. These miRNA family members have been linked to several tumors and other chronic degenerative diseases, playing a role as tumor suppressors or oncogenes (7-9).

In the present study, we showed that miR-125a is downregulated in patients with $\mathrm{CC}$ who do not respond to standard chemotherapy and radiotherapy treatment. Then, we employed a radioresistance in vitro model employing $\mathrm{SiHa}, \mathrm{CaSki}$ and HeLa cell lines established by fractionated radiation in order to elucidate the role of miR-125 in the induction of radioresistance. Finally, we demonstrated that overexpression of miR-125a significantly decreased radioresistance through the negative regulation of CDKN1A in CC cells.

\section{Materials and methods}

Patient selection and CC samples. We selected 62 tumor samples from the National Cancer Institute of Mexico (INCan) Tumor Bank; 30 fresh-frozen cancer samples were analyzed for miR-125a expression by means of qRT-PCR (15 patients were non-responders and 15, complete responders). Additionally, 32-paraffin-embedded tissues were employed to assess p21 expression by immunohistochemistry (IHC) analysis. All patients included accepted to participate in the study and signed informed consent; the Institutional Ethics and Scientific Board Committees approved the protocol in accordance with The Code of Ethics of the World Medical Association (WMA) (Declaration of Helsinki). All patients were histologically and clinically diagnosed with locally advanced CC [stages IB2-IVA according to the International Federation of Gynecology and Obstetrics (FIGO) classification]. Samples were obtained from patients diagnosed between 2011 and 2014 at the Department of Obstetrics and Gynecology, INCan. All patients had a median of 55 months of clinical follow-up. After sample-taking, the patient samples were categorized into 2 groups depending on their clinical response to standard treatment. Complete response (CR) was defined as the disappearance of all signs of cancer in response to treatment, and no response (NR) was defined as patients with partial, progressive or stable disease. The tumor samples employed in the present study contained at least $80 \%$ of tumor cells on pathological examination. Standard treatment for patients with locally advanced CC consists of 5 cycles of $40 \mathrm{mg} / \mathrm{m}^{2}$ of CDDP (cis-diamminedichloroplatinum II) and a total of 55 Gy of radiotherapy and 30 Gy of internal brachytherapy.

Cell lines. Human CC cell lines SiHa (HTB-35), CaSki (CRL-1550) and HeLa (CCL-2) were purchased from the American Type Culture Collection (ATCC; Rockville, MD, USA) and cultured according to cell-line specifications. CaSki and HeLa cell lines were cultured in RPMI-1640 medium, although the SiHa cell line was cultured in EMEM medium. All cell lines were maintained with $100 \mathrm{U} / \mathrm{ml}$ of penicillin and
$100 \mathrm{mg} / \mathrm{ml}$ of streptomycin, $10 \%$ fetal bovine serum, and incubated at $37^{\circ} \mathrm{C}$ in a $5 \% \mathrm{CO}_{2}$ atmosphere. All cancer cell lines employed in the present study were authenticated by means of the Authentifiler PCR Amplification kit (cat. no. 4479566; Thermo Fisher Scientific, Inc., Waltham, MA, USA) on a 3500 Genetic Analyzer (cat. no. 4440462; Applied Biosystems, Foster City, CA, USA) following the International Cell Line Authentication Committee (ICLAC) guidelines.

Establishment of a radioresistant in vitro model. The human $\mathrm{CC}$ cell lines $\mathrm{SiHa}, \mathrm{CaSki}$ and HeLa were employed to establish a radioresistant cell line model by fractionated radiation. The parental cell lines were grown to $80 \%$ confluence, and then the cell lines were trypsinized and divided into 2 subcultures: one for irradiation (RR, radioresistant cells), and the remaining subculture for the non-irradiated condition ( $\mathrm{RS}$, radiosensitive cells). When RR culture cells reached $60 \%$ confluence, they were irradiated with 2 Gy of X-ray irradiation using an X-ray Linear Accelerator (CL2100C/D; Varian Medical Systems, Palo Alto, CA, USA); immediately after irradiation, the cells were returned to the incubator. Then, after $24 \mathrm{~h}$, the irradiated cell lines were trypsinized and subcultured into new flasks. When they again reached $60 \%$ confluence, the irradiation protocol was repeated until the cells reached a total dose of 56 Gy. RS cells were treated under the same conditions as the $\mathrm{RR}$ culture, but without irradiation.

Determination of lethal dose 50 of radiation. Lethal dose 50 $\left(\mathrm{LD}_{50}\right)$ was determined by colony formation assay (CFA). The parental cell lines were incubated at $37^{\circ} \mathrm{C}$ for $24 \mathrm{~h}$ and subsequently were irradiated at different doses ranging from 0-10 Gy. The cells were harvested and counted $24 \mathrm{~h}$ after irradiation. Subsequently, $3 \times 10^{3}$ cells were plated in 6-well culture plates and incubated under standard conditions for 2 weeks. The colonies formed were fixed and stained with glutaraldehyde $6.0 \%$ ( $\mathrm{vol} / \mathrm{vol})$ and crystal violet $0.5 \%$ (wt/vol) in water. Finally, colonies consisting of 50 cells or more were counted using an optical microscope and the surviving fraction was determined.

RNA isolation from tumor samples and cell lines. Total RNA from CC tissues and cell lines were extracted and purified with the miRNeasy Mini kit (cat. no. 217004; Qiagen, Inc., Valencia, CA, USA) according to the manufacturer's instructions. RNA quantification was performed using an Epoch spectrophotometer (BioTek Instruments, Inc., Winooski, VT, USA).

Relative quantification of miR-125 by qRT-PCR. The expression of miR-125 was assessed using the TaqMan MicroRNA assay (Applied Biosystems, Carlsbad, CA, USA) according to the manufacturer's protocol. Briefly, $100 \mathrm{ng}$ of total RNA was subjected to reverse transcription reaction using miRNA-specific RT primers and the TaqMan miRNA reverse transcription kit. The $15-\mu 1$ reactions were incubated according to the manufacturer's protocol. Real-time qPCR was performed using TaqMan Universal Master Mix II no UNG in a StepOne qPCR instrument (Applied Biosystems, Carlsbad, CA, USA). Relative expression of miR-125a was calculated utilizing the comparative $2^{-\Delta \Delta C t}$ method. RNU-44 and RNU-6b expression were employed as endogenous control. All of the 
qPCR reactions were assessed in 3 independent experiments and each reaction was performed in triplicate.

Western blot analysis. Total protein from cell lysates was extracted using RIPA buffer (sc-24948; Santa Cruz Biotechnology, Inc., Santa Cruz, TX, USA). Then, $50 \mu \mathrm{g}$ of protein was separated by SDS-polyacrylamide gel electrophoresis (PAGE) and transferred onto a polyvinylidene difluoride (PVDF) membrane (GE Healthcare, Milwaukee, WI, USA) in a Trans-Blot Turbo (Bio-Rad) semi-dry chamber at $25 \mathrm{~V}$ and $1 \mathrm{~mA}$ for $30 \mathrm{~min}$. After blocking with $5 \%$ non-fat milk for $2 \mathrm{~h}$, the membrane was incubated with the specific antibody overnight at $4^{\circ} \mathrm{C}$ on a rocking platform, washed, and then incubated with the corresponding secondary antibody for $2 \mathrm{~h}$ at room temperature. The blot was visualized using the Super Signal West Femto Chemiluminescent substrate (Pierce, Rockford, IL, USA) in a C-Digit scanner (LI-COR) ${ }^{\mathrm{TM}}$ employing Image Studio (LI-COR Biosciences, Lincoln, NE, USA) software. The primary antibodies were purchased from Santa Cruz Biotechnology, Inc.: anti-p21 (187) (1:2,000; sc-817). All secondary antibodies were obtained from the Cell Signaling Technology, Inc. (Beverly, MA, USA); anti-mouse (1:5,000; \#7076S). $\beta$-actin (C4) (1:5,000; sc-47778; Santa Cruz Biotechnology, Inc.) was utilized as an internal control.

Luciferase reporter assays. Reporter plasmids were constructed by ligation of synthetic oligonucleotide duplexes (IDT) containing one of the 3 putative miR-125a target regions in the CKN1A mRNA 3'UTR, including region 1, 5'-CTAGTG CACTGGGGAGCCCGTCTCAGTGTA-3' and AGCTTA CACTGAGACGGGCTCCCCAGTGCA; region 2, 5'-CTA GTACACAAGGGCACCCTAGTTCTACCTCAGGCAA-3'; and region 5'-AGCTTTGCCTGAGGTAGAACTAGG'-GTG CCCTTCTTGTGTA-3'; and finally, region 3, 5'-CTAGTA GACTGTAAACCTCTCGAGGGCA-3'; and region, 5'-AGC TTGCCCTCGAGAGGTTTACAGTCTA-3'. All putative regions were obtained from microRNA.org and cloned into the pMIR-REPORT plasmid (Ambion Inc., Austin, TX, USA) (10). Each construction was co-transfected with miR-125a mirVana miRNA mimic (Applied Biosystems) and the pMIR-REPORT $\beta$-gal control plasmid (Ambion) into HeLa cells. Luciferase activity was analyzed $48 \mathrm{~h}$ after transfection utilizing the Dual-Luciferase Reporter Assay System (Applied Biosystems) in a GloMax 96 Microplate Luminometer (Promega, Madison, WI, USA). Luciferase activity was normalized to $\beta$-gal activity for each transfected well; each experiment was performed in triplicate.

Transfection of miRNA mimics and inhibitors. miR-125a mimics and inhibitors were purchased from Ambion and assessed according to the manufacturer's instructions. The pre-miR negative control and scramble oligonucleotide for the miRNA transfection experiments were not homologous to any human miRNA sequences and can be obtained from the Pre-miR miRNA Starter kit (cat. no. Am1540; Thermo Fisher Scientific, Inc.). Oligonucleotide transfection was performed using the Lipofectamine RNAiMAX transfection reagent (cat. no. 13778150; Thermo Fisher Scientific, Inc.). All experiments were replicated in 6-well plates with a final concentration of $25 \mathrm{pmol}$ of each oligonucleotide,
Table I. Clinical and pathological characteristics of the cervical cancer patients enrolled in the present study

\begin{tabular}{|c|c|}
\hline Characteristics & Patients, n (\% \\
\hline Total number & 62 \\
\hline \multicolumn{2}{|l|}{ Age (years) } \\
\hline Median & 52 \\
\hline Range & $31-68$ \\
\hline \multicolumn{2}{|l|}{ Histological type } \\
\hline Squamous cell carcinoma & $54(87.1)$ \\
\hline Adenocarcinoma & $8(12.9)$ \\
\hline \multicolumn{2}{|l|}{ Tumor size $(\mathrm{cm})$} \\
\hline$<4$ & $11(17.8)$ \\
\hline$\geq 4$ & $51(82.2)$ \\
\hline Without data & $0(0.0)$ \\
\hline \multicolumn{2}{|l|}{ Clinical stage (FIGO) } \\
\hline IIA & $0(0.0)$ \\
\hline IIB & $37(59.9)$ \\
\hline IIIA & $16(25.6)$ \\
\hline IIIB & $9(14.5)$ \\
\hline IVA & $0(0.0)$ \\
\hline \multicolumn{2}{|l|}{ HPV genotypification } \\
\hline Type 16 & $29(46.8)$ \\
\hline Type 18 & $16(25.8)$ \\
\hline Type 45 & $15(24.2)$ \\
\hline Others & $2(3.2)$ \\
\hline \multicolumn{2}{|l|}{ Clinical response } \\
\hline Complete response & $32(51.6)$ \\
\hline No response & $30(48.4)$ \\
\hline
\end{tabular}

FIGO, International Federation of Gynecology and Obstetrics.

and $7.5 \mu \mathrm{l}$ of Lipofectamine RNAi/MAX was used for each transfection.

P21 expression in CC tissues. IHC was performed on serial sections using p21 (187) (sc-817; Santa Cruz Biotechnology, Inc.) antibody in 32 paraffin-embedded blocks from patients with CC (non-responders, 15; and complete responders, 17). Serial sections, $5-\mu \mathrm{m}$ thick, were immunostained using the biotin-streptavidin-peroxidase method. Briefly, the sections were deparaffinized in xylene and rehydrated with ethanol. Then, the samples were hydrated by autoclave pretreatment in $10 \mathrm{mM}$ citrate buffer ( $\mathrm{pH}$ 6.0) for $5 \mathrm{~min}$. Endogen peroxidase was quenched with $3 \%$ hydrogen peroxidase for $10 \mathrm{~min}$ at room temperature. Next, the slides were incubated with the primary antibody at a 1:200 dilution in Tris-buffered solution (50 mM Tris- $\mathrm{HCl}, 150 \mathrm{mM} \mathrm{NaCl}, \mathrm{pH}$ 7.4) for $2 \mathrm{~h}$. Finally, the antibody was visualized with 3,3-diaminobenzidine tetrahydrochloride. The fraction of positive cells was estimated using a four-tiered scale ( $\leq 5 \%, 1 ; 6-25 \%, 2 ; 51-75 \%, 3$ and $\geq 75 \%, 4)$. Staining intensity was also scored on a 4-tiered scale (negative, 0 ; low-intensity positive staining, 1 ; moderate-intensity positive staining, 2; and strong-intensity positive staining, 3 . 

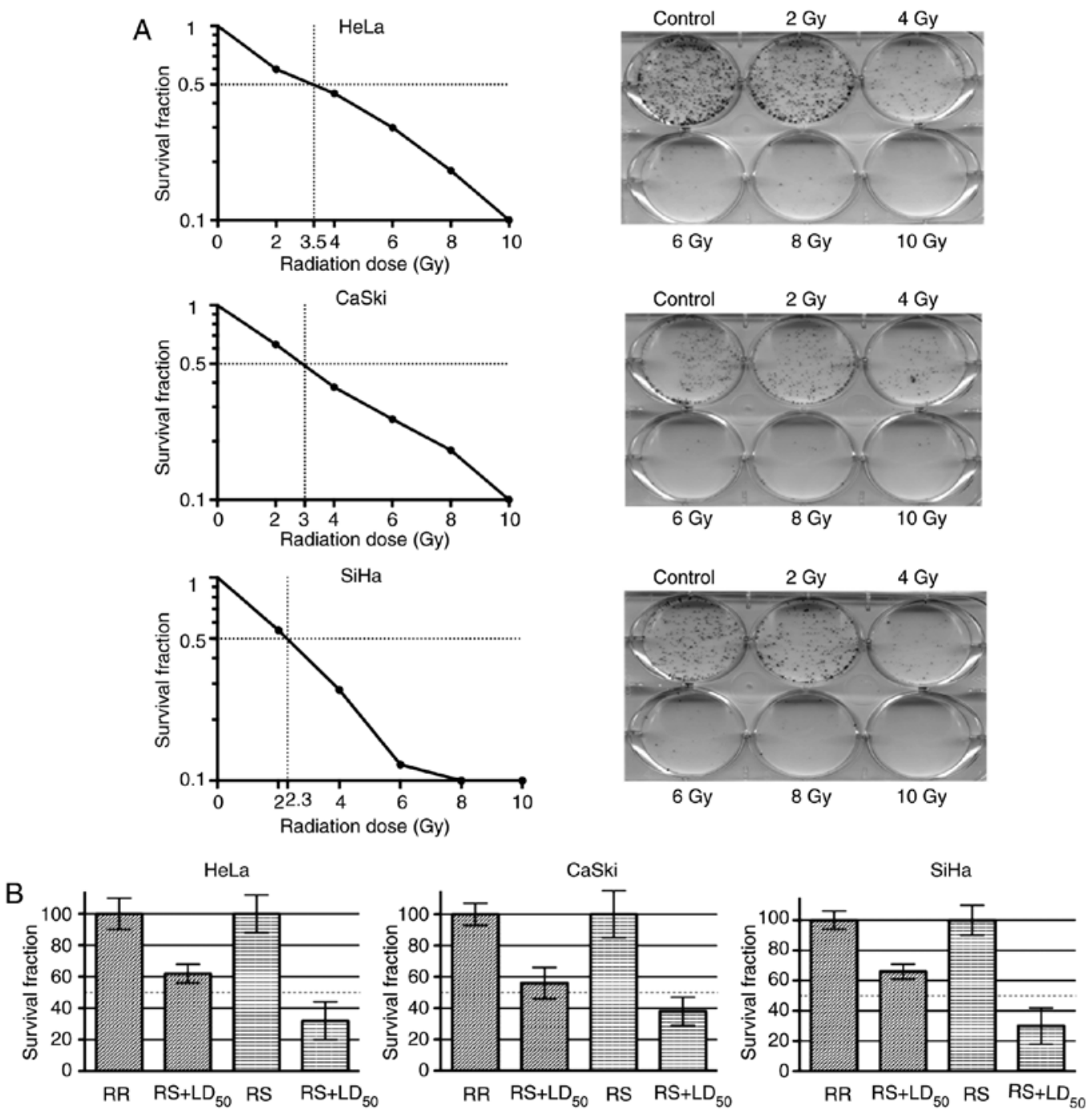

Figure 1. Radioresistant-induced model in HeLa, CaSki and SiHa cell lines. (A) Cell lines were irradiated at different doses ranging from 0-10 Gy. After 2 weeks of incubation, $\mathrm{LD}_{50}$ was determined by colony formation assay, resulting in a specific $\mathrm{LD}_{50}$ for each cell line as follows; CaSki=3 Gy; HeLa=3.5 Gy, and $\mathrm{SiHa}=2.3 \mathrm{~Gy}$. (B) Parental and radioresistant cell lines were irradiated once with $\mathrm{LD}_{50}$ calculated doses to confirm the radioresistant phenotype. The survival fraction of HeLa-RR, SiHa-RR and CaSki-RR were 58, 62 and 64\%, respectively. As expected, all of the RR subcultures had a higher survival rate than RS cultures.

Two independent pathologists evaluated the stained sections and the average score for each slide was used for statistical analysis.

Statistical analysis. Data are expressed as the mean \pm standard error of the mean (SEM) of at least 3 separate experiments performed in triplicate. The differences between groups were analyzed using a double-sided Student's t-test when only 2 groups were present, and the null hypothesis was rejected at 0.01 levels unless otherwise specified.

\section{Results}

Radioresistant cell model. We established a cancer cell line radioresistant model by fractionated irradiation. First, we determined the $\mathrm{LD}_{50}$ of radiation for HeLa, CaSki and SiHa cell lines by CFA. The previously mentioned cell lines were irradiated at different doses $(0-10 \mathrm{~Gy})$ and survival curves were determined. Therefore, we calculated a 'parental' $\mathrm{LD}_{50}$ for each cell line. Hence, HeLa cells exhibited a $\mathrm{LD}_{50}$ higher than that of the CaSki and SiHa cells (CaSki, 3.0 Gy; HeLa, 3.5 Gy; and $\mathrm{SiHa}, 2.3 \mathrm{~Gy}$ ) (Fig. 1A).

After 28 episodes of irradiation, cell lines HeLa-RR, SiHa-RR and CaSki-RR reached a total of $56 \mathrm{~Gy}$, and stable radioresistant cells were obtained from the surviving fraction of parental irradiated cells. Next, $\mathrm{LD}_{50}$ for parental cell lines were employed to confirm the radioresistant phenotype in the irradiated cell lines. After parental cell lines were irradiated at $\mathrm{LD}_{50}$-calculated doses, as expected, all of the RR subcultures had a higher survival rate than the RS cultures. Accordingly, the survival fractions of the HeLa-RR, SiHa-RR and CaSki-RR cells were 58, 62 and 64\%, respectively (Fig. 1B). Therefore, we confirmed the establishment of a radioresistant phenotype in the RR subcultures.

CC samples. The patients with CC enrolled in the present study had a clinical and pathological diagnosis of locally advanced CC (LACC). All cervical samples were histologically analyzed to confirm a minimum of $80 \%$ tumor cells. A summary of the clinical and pathological characteristics of 

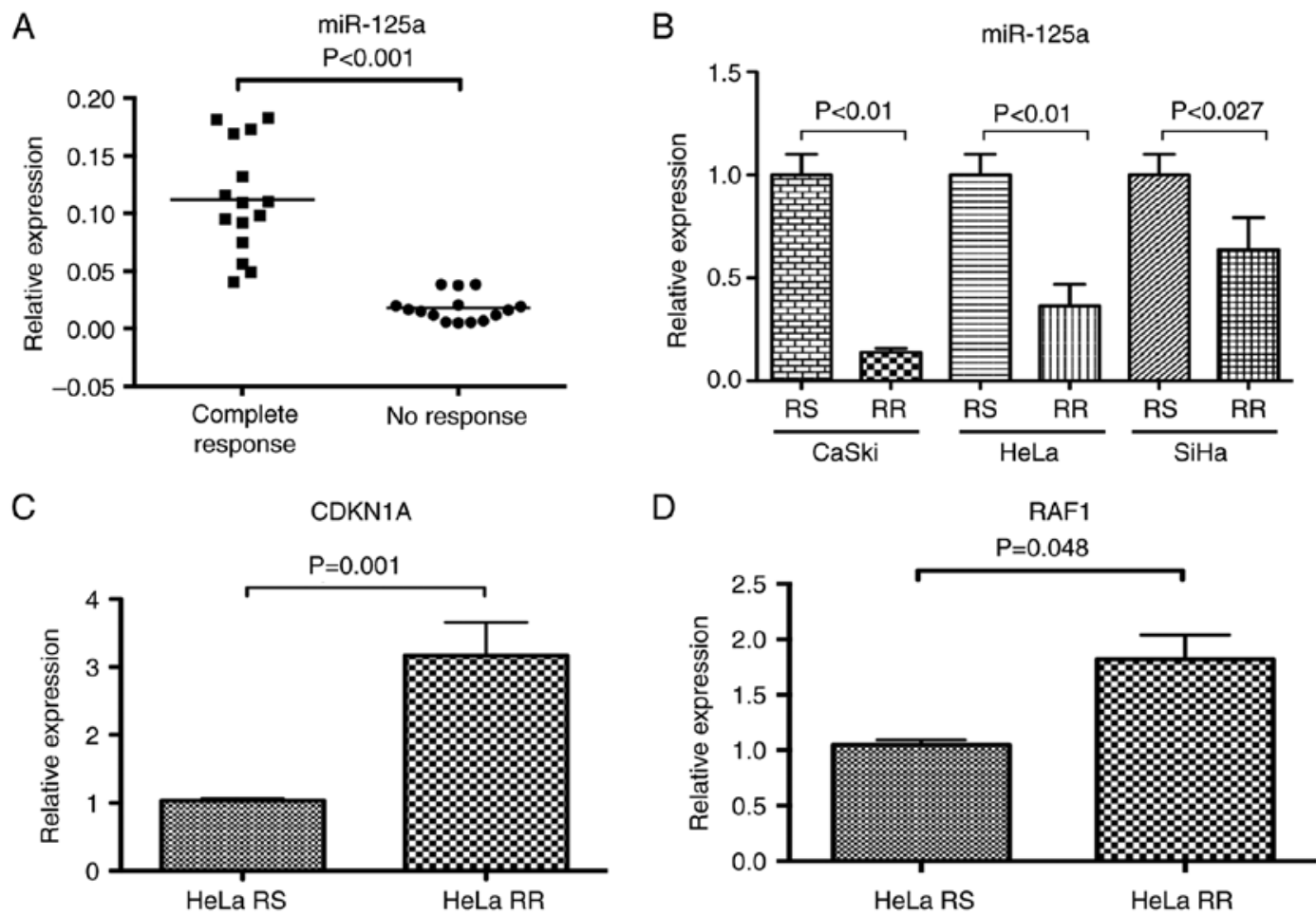

Figure 2. Relative expression of miR-125a, CDKN1A and RAF1. (A) Relative expression of miR-125a was analyzed in 30 patients with CC [complete response $(\mathrm{CR})=15$, and no response $(\mathrm{NR})=15]$. Each dot represents the relative expression level for a single patient by triplicate. Relative expression was normalized with RNA-U6- and RNU-44-level expression. (B) Relative expression of miR-125a was evaluated in CaSki, HeLa and SiHa cells lines. miR-125 was downregulated in CaSki ( $\mathrm{P}=0.0001)$, HeLa ( $\mathrm{P}=0.001)$ and $\mathrm{SiHa}(\mathrm{P}=0.027)$ cell lines. (C) CDKN1A, a molecular target of miR-125a, was upregulated in the HeLa-RR cell line, with respect to the HeLa-RS cell line $(\mathrm{P}=0.01)$. (D) RAF1, a molecular target of miR-125a, was upregulated in the HeLa-RR cell line with respect to the HeLa-RS cell line $(\mathrm{P}=0.048)$.

all patients is presented in Table I. Median age of patients at diagnosis was 52 years (range, 31-68 years). As expected, the most prevalent HPV genotypes identified were HPV-16 (46.8\%), HPV-18 (25.8\%) and HPV-45 (24.2\%).

Relative quantification of miR-125a in CC samples and the radioresistant cell model. We recently published an expression profile of miRNA associated with clinical response in patients with $\mathrm{CC}$ under standard treatment of chemotherapy and radiotherapy; one of these identified miRNA was miR-125a (6). Based on this evidence, we aimed to elucidate the functional role of miR-125a in the radioresistance phenotype in $\mathrm{CC}$. Hence, we assessed the expression level of miR-125a in 30 dichotomized CC samples ( $\mathrm{NR}=15$ and $\mathrm{CR}=15$ ). Relative expression of miR-125a was significantly lower in the $15 \mathrm{NR}$ samples with regard to the CR samples ( $\mathrm{P} \leq 0.0001)$ (Fig. 2A).

We quantified the expression level of miR-125a in HeLa-RR, SiHa-RR and CaSki-RR subcultures (Fig. 2B). miR-125a was underexpressed in all RR subcultures with respect to RS cell lines.

It is noteworthy that miR-125a was found 5-fold underexpressed in the CaSki RR cell line with respect to the CaSki RS cell line ( $\mathrm{P}=0.0001)$, whereas in HeLa-RR and SiHa-RR cell lines, miR-125a was found up to 2.5 -fold underexpressed $(\mathrm{P}=0.001$ and $\mathrm{P}=0.027$, respectively). We conducted the following experiments on the HeLa cell line in order to achieve a better performance of transfection assays, such as that reported by Asgharian et al (11).

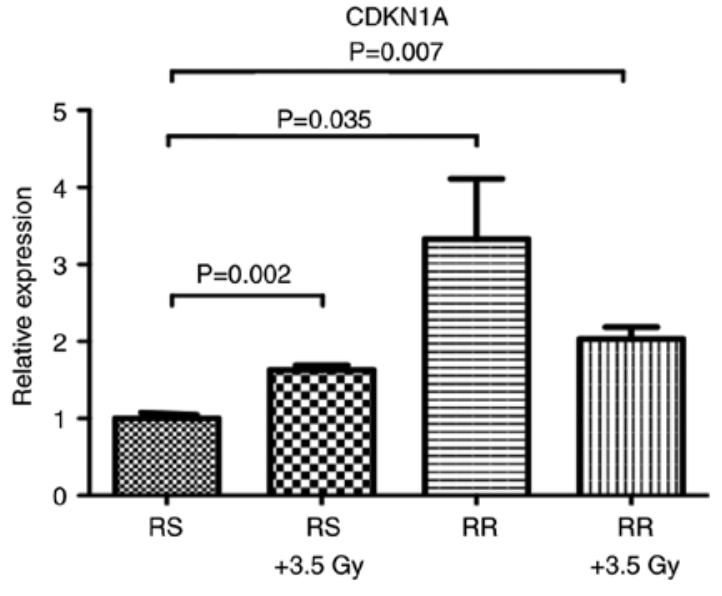

Figure 3. Relative expression of CDKN1A in HeLa-RR and HeLa-RS cell lines. (A) Relative expression of CDKN1A was evaluated in a radioresistant (RR) HeLa cell line with regard to radiosensitive (RS) parental cells. The CDKN1A gene was upregulated in the HeLa-RR cell line $(\mathrm{P}=0.035)$ Additionally, the CDKN1A gene was deregulated when the HeLa-RS cell line was irradiated $(\mathrm{P}=0.002)$.

Molecular targets of miR-125a. After confirming that miR-125a was underexpressed in resistant CC samples and radioresistant (RR) CC cell lines, we conducted an exhaustive search on the most citable bioinformatics algorithms for predicting miR-125a targeted mRNA (microRNA.org, TargetScan and miRDB). Five hypothetical target genes of miR-125 were obtained by means of this method (CDKN1A, SP1, E2F7, 
A
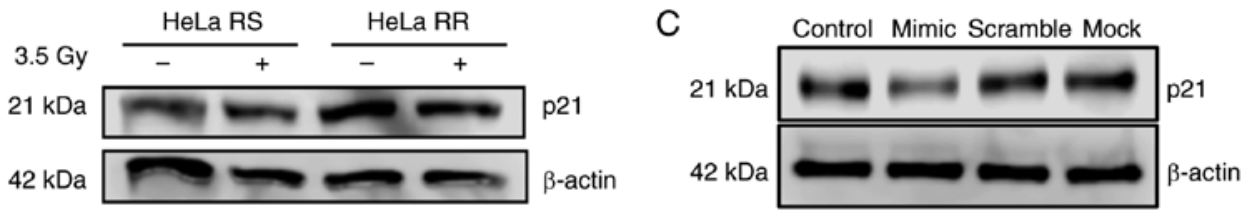

B
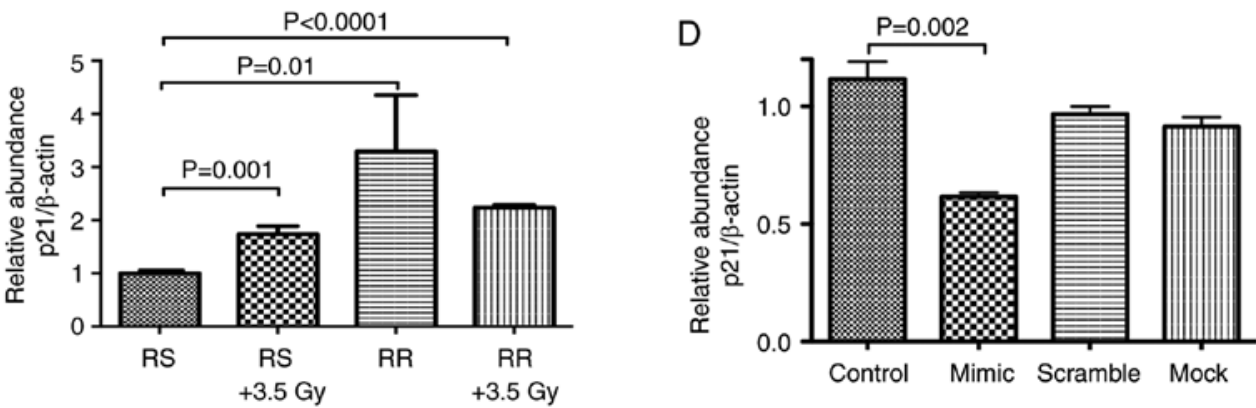

Figure 4. p21 is upregulated in the HeLa-RR cell line. p21 protein level was upregulated up to 4-fold in the HeLa-RR cell line in respect to the HeLa-RS cell line $(\mathrm{P}=0.0194)$. After $24 \mathrm{~h}$ of irradiation with $3.5 \mathrm{~Gy}$, HeLa-RS cells exhibited a 2 -fold increase in p21 protein expression $(\mathrm{P}=0.0011)$ : $\beta$-actin was used as a loading control. (A) Western blotting (WB) in HeLa-RS and HeLa-RR cells with/without single-dose radiation. (B) Densitometry analysis. (C) HeLa cells were transfected with miR-125 mimic, negative control, scramble molecule or mock condition. After $48 \mathrm{~h}$ of transfection, cell lysates were obtained for WB analysis of p21, $\beta$-actin was used as a loading control. (D) Restoration of miR-125a expression decreased the protein levels of p21 with respect to the control transfection condition $(\mathrm{P}=0.002)$.

A

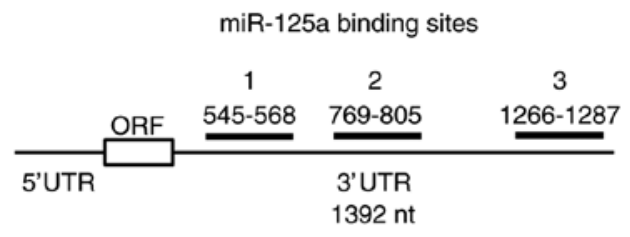

p21 transcript

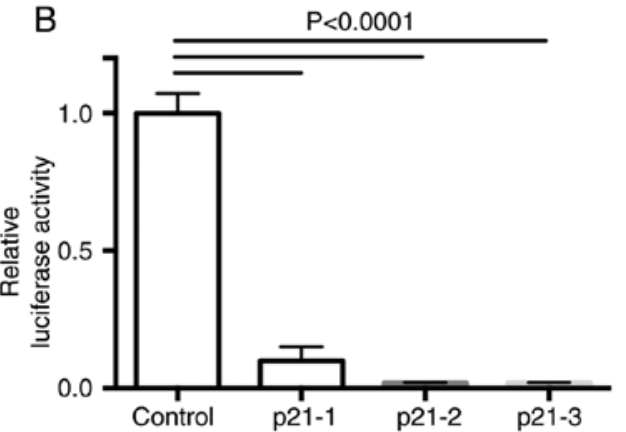

C

\begin{tabular}{|c|c|c|c|}
\cline { 2 - 3 } \multicolumn{1}{c|}{} & \multicolumn{2}{c|}{ miR-125a expression } & \multirow{2}{*}{ P-value } \\
\cline { 2 - 3 } & $\begin{array}{c}\text { High expression } \\
n=17\end{array}$ & $\begin{array}{c}\text { Low expression } \\
n=15\end{array}$ & \\
\hline & & & \\
p-21 expression & $9(52.94)$ & $9(60.0)$ & 0.68 \\
Negative $(n=18)$ & $8(47.06)$ & $6(40.0)$ & \\
Positive $(n=14)$ & & & \\
& & & \\
\hline
\end{tabular}

Figure 5. CDKN1A is downregulated by miR-125a in the CC cell line. (A) Sequence context of the 3 binding sites into the 3'UTR of the CDKN1A gene. The localization of the 3 binding sites that were cloned were the following: 1, 545-568 nt; 2, 769-805 nt; and 3, 1266-1287. (B) Luciferase reporter constructions containing one of the 3 binding sites of p21 were co-transfected with miR-125a mimics into HeLa cells. All 3 binding sites showed a dramatic decrease in luciferase activity. Luciferase reporter assays were assessed 3 times and data are presented as mean \pm SD. (C) Thirty-two cervical CC tissues were immunohistochemically stained with p21 antibody and dichotomized according to their miR-125a expression level. The Fisher's exact test was used to corroborate statistical associations.

AKT1 and RAF1), which were tested by qRT-PCR to suggest a possible regulation by miR-125a. We found an overexpression of CDKN1A and RAF1 (Fig. 2C and D) transcripts in the HeLa cell line $(\mathrm{P}=0.01$ and $\mathrm{P}=0.048)$, respectively, whereas, differences in SP1, E2F7 and AKT1 mRNA were not statistically significant (data not shown). Additionally, we demonstrated that CDKN1A is overexpressed after $\mathrm{LD}_{50}$ irradiation doses, in both RS and RR cells (Fig. 3), suggesting a possible role of CDKN1A in the radioresistant phenotype.
Western blot analysis of p21. The protein levels of p21 (CDKN1A) were assessed in the HeLa-RS and HeLa-RR cells. Our results confirmed that $\mathrm{p} 21$ was overexpressed by up to 3 -fold in the HeLa-RR cells with regard to the HeLa RS cells $(\mathrm{P}=0.019)$. In addition, the radiation increased $\mathrm{p} 21$ protein levels 2 -fold in the RS cells $(\mathrm{P}=0.001)$. It was encouraging to note that in all cases where the cells were irradiated, p21 was overexpressed (Fig. 4A and B). RR cells had higher levels of CDKN1A, probably due to a downregulation in the expression 
A
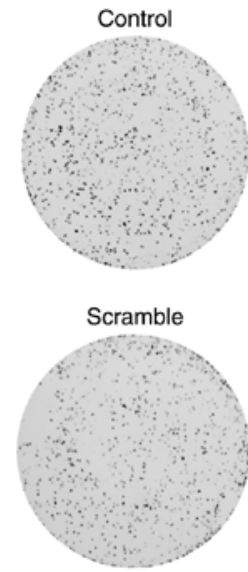

B

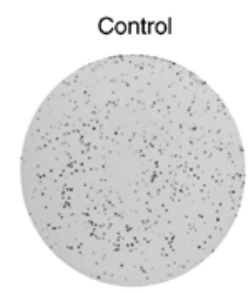

Scramble

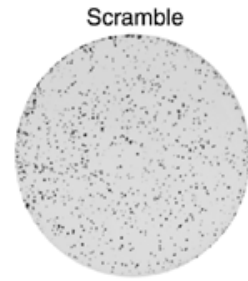

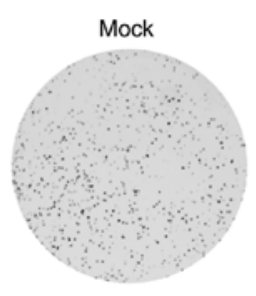
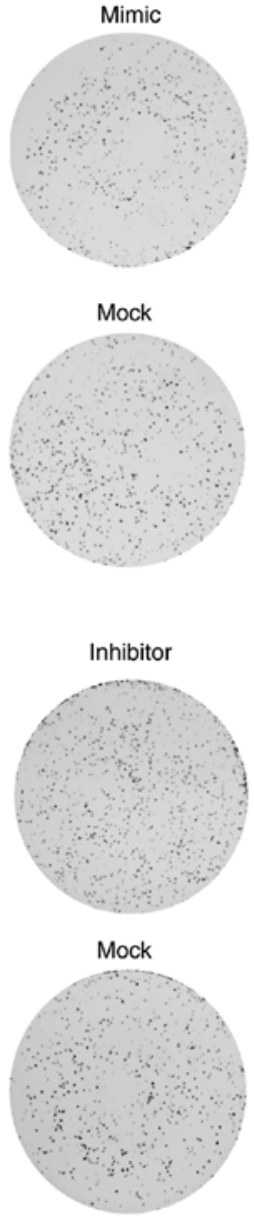
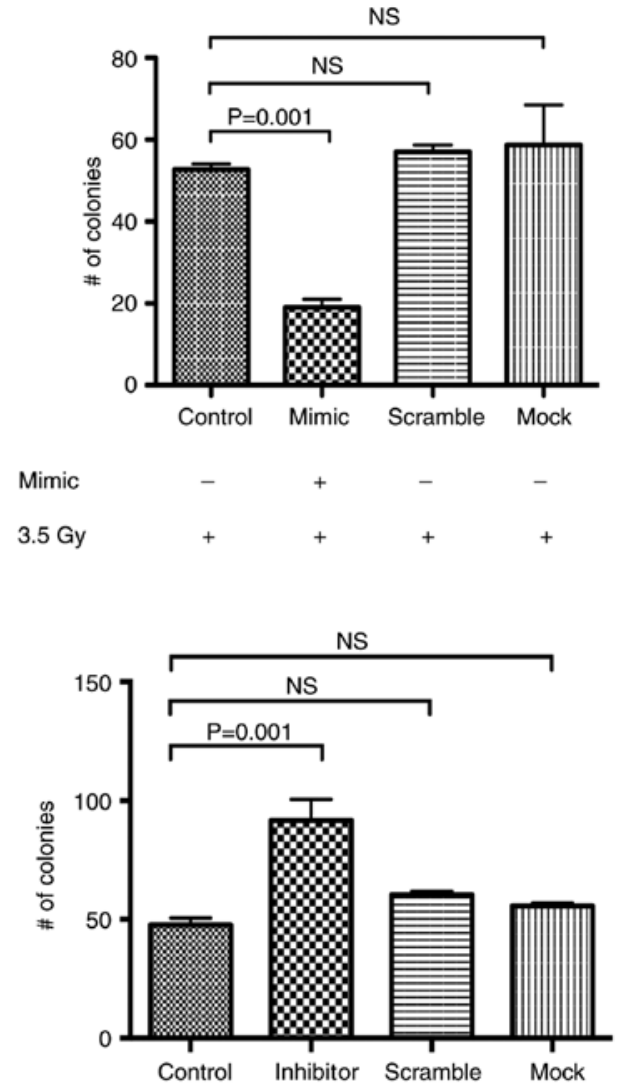

Inhibitor

3.5 Gy

Figure 6. miR-125a sensitizes cervical cancer cells to radiation therapy. (A) HeLa cells were transfected with 25 pmol of miR-125a mimics $48 \mathrm{~h}$ prior to being irradiated with a single dose of $3.5 \mathrm{~Gy}$. miR-125a mimic-transfected cells demonstrated significantly lower colony survival rates than control cells. (B) HeLa cells were transfected with 25 pmol of miR-125a inhibitor $48 \mathrm{~h}$ before being irradiated with a single dose of 3.5 Gy. miR-125a inhibitor-transfected cells showed significantly higher colony survival rates than the control cells. NS, not significant.

of miR-125a. In order to test this hypothesis, we restored the expression of miR-125a by means of a mimic sequence transfected into RR cells. As observed in Fig. 4C and D, restoration of miR-125a by a mimic sequence decreased the protein levels of p21, suggesting a direct role for CDKN1A regulation exerted by miR-125a.

miR-125a regulates the expression of CDKN1A. To validate whether CDKN1A mRNA is a molecular target of miR-125a, we performed a luciferase reporter assay. Three putative binding sites for miR-125a in the CDKN1A 3'UTR were cloned into the pMIR-REPORT plasmid. The binding regions between miR-125a and CDKN1A mRNA comprise the following: $1,545-568 ; 2,769-805$, and 3, 1266-1287 nucleotides into the 3 'UTR of the p21 transcript (Fig. 5A). After normalization with the $\beta$-gal control, luciferase activity was suppressed in all 3 binding sites cloned. These results demonstrated that the 3 cloned miR-125a-binding sequences are usable for inhibition of p21 transcript expression by miR-125a (Fig. 5B).

P-21 expression in CC tissues. In order to confirm that $\mathrm{p}-21$ could be a RR marker in CC tissues; we assessed protein expression by means of IHC in 32 paraffin-embedded tissues (in $15 \mathrm{CR}$ and in $17 \mathrm{NR}$ tissue samples). We observed a slight difference in the $\mathrm{p} 21$ protein expression level between both sample groups without a statistically significant significance (Fig. 5C). We hypothesized that the number of samples analyzed by IHC did not allow corroborating a higher level of the p21 protein in patients diagnosed as non-responders to standard chemotherapy and radiotherapy treatment.

miR-125a sensitizes $C C$ cell lines to radiation therapy. To elucidate whether overexpression of miR-125a sensitizes CC cells to irradiation treatment, we transfected miR-125a mimics and inhibitors into HeLa cells and determined the sensitivity of transfected cells after the irradiation dose. First, we corroborated the level of expression of p21 in the transfected cells (Fig. 4C). Then, after $24 \mathrm{~h}$, we performed a post-radiation CFA and cell survival fractions were calculated after 2 weeks of incubation. Notably, we found a significant decrease in survival fractions in the miR-125 mimic-transfected cells (Fig. 6A) compared to their control cell lines (negative and scramble oligonucleotide conditions) $(\mathrm{P}=0.005)$. When cells were transfected with the miR-125 inhibitor we found a higher number of colonies (Fig. 6B; $\mathrm{P}=0.001$ ) in respect to their respective negative and scramble control cells. These findings strongly suggest that miR-125a is a regulator of acquired radioresistance in CC. 


\section{Discussion}

Recently, several research groups have reported that the radioresistance phenotype in cell lines and tumor samples could be explained by the expression profile of miRNAs. Notably, these studies have listed some miRNAs as possible regulators of the radioresistant phenotype (12-14). However, only a few of these have been assessed by functional assays to demonstrate a possible regulatory role. In the present study, we demonstrated that miR-125a regulates the expression of $\mathrm{p} 21$ and that this event is related to the sensitization of CC cells to radiation therapy. The acquisition of radioresistance is a complex process in which a wide number of genes and several signaling pathways are involved (15-17). A single miRNA regulates several hundreds of genes that could be implicated in the radioresistance phenotype. Due to this, miRNAs possess evident advantages for their consideration as radioresistance modulators based on their ability to control multiple targets in specific molecular pathways.

Here we showed that miR-125a is downregulated in patients with cervical cancer diagnosed as non-responders (NRs) to conventional therapy (Fig. 2A). Moreover, these results were validated in vitro employing radioresistant (RR) and radiosensitive (RS) HeLa, SiHa and CaSki cervical cancer cell lines (Fig. 2B). This evidence indicates a correlation between the expression level of miR-125a and the radioresistant phenotype in cervical cancer.

Recently, Moskwa et al identified a subset of miRNAs associated with radioresistance in glioblastoma cell lines. Among the identified miRNAs there were the following: miR-1; miR-150; miR-425; and notably, miR-125a (18). In addition, the authors demonstrated, by functional assays, that miR-125a promotes radioresistance in LN229 and U251 cell lines. In this same research line, Shiiba et al demonstrated the participation of miR-125b (an miR-125a homologous miRNA) in the acquisition of radioresistance in oral squamous cell carcinoma cells to radiation therapy (19). In CC, Fan et al showed that downregulation of miR-125a is correlated with preoperative metastasis and poorer progression-free survival (PFS) and overall survival (OS) rates in patients with CC (20). In a subsequent study published by Fan $e t a l$, the authors demonstrated that miR-125a is one of the most significantly subexpressed miRNAs in HeLa and CaSki CC cell lines resistant to cisplatin. Through functional assays, they demonstrated that miR-125a depletion sensitizes chemoresistant CC cell lines to paclitaxel in vitro and in vivo, and to cisplatin in vivo through the regulation of STAT3 (21). These previously published data strongly support our findings that the downregulation of miR-125a is associated with non-response to conventional treatment in patients with $\mathrm{CC}$ and could be involved in the regulation of radioresistance and chemoresistance in $\mathrm{CC}$ cell lines.

CDKN1A is a validated target of miR-125a; in the present study we showed that CDKN1A is overexpressed by radioresistant cervical cancer cell lines. The CDKN1A gene is located on chromosome 6p21.2 and encodes for the cyclin-dependent kinase inhibitor 1 protein, better known as p21. Depending on its cellular localization, p21 engages in several functions. Hence, nuclear p21 inhibits the activity of cyclin-dependent kinases Cdk1 and Cdk2, blocking G1/S transition. Cytoplasmic p21 inhibits the function of caspase-3, accomplishing anti-apoptotic functions. Various studies have shown that p21 is deregulated by the effect of ionizing radiation and is associated with the acquisition of radioresistance $(22,23)$. In the present study, we demonstrated that the p21 protein is overexpressed in radioresistance cell lines, confirming previous studies (Fig. 4A and B).

Additionally, we noted that CDKN1A mRNA and p21 protein expression is upregulated when the cells are exposed to a sublethal radiation dose ( $3.5 \mathrm{~Gy}$ ). Taken together, our results showed that the expression of miR-125a is associated with the response to radioresistance in patients with $\mathrm{CC}$ and, additionally, they are correlated the decreased expression of p21 in radioresistant cells. To corroborate the aforementioned findings, we demonstrated that the ectopic expression of miR-125 decreased $>40 \%$ of the expression of the p21 protein in HeLa cells $48 \mathrm{~h}$ post-transfection, and that this severely affects its cellular ability to respond to radiation therapy (Fig. 6A and B).

The identification of specific miRNAs associated with the regulation of radioresistance may lead to improved, efficient treatments for patients with CC. In this respect, new experimental methodologies should be able to corroborate the role of miRNA candidates through functional assays. Moreover, best-candidate miRNAs should be able to be detected in serum fractions as useful predictor biomarkers of the radioresistance phenotype. As demonstrated in the present study, the miR-125a mimic sensitizes cervical cancer cells to radiation therapy, and regulation of their target genes directly impacts the molecular mechanisms associated with the acquisition of radioresistance.

These findings and those previously published by other authors support the idea that miRNAs could function as central regulators of radioresistance acquisition by cancer cells. Our findings determined a specific role for miRNA-125a in the radioresistance of cervical cancer, and it may be considered for future therapeutic strategies for this neoplasia.

\section{Acknowledgements}

The present study was partially supported by the CONACyT (SALUD-2014-1-233733 and Cátedras-2425).

\section{References}

1. Torre LA, Bray F, Siegel RL, Ferlay J, Lortet-Tieulent J and Jemal A: Global cancerstatistics, 2012. CA Cancer J Clin. Mar 65: 87-108, 2015.

2. Delaney G, Jacob S, Featherstone C and Barton M: The role of radiotherapy in cancer treatment: Estimating optimal utilization from a review of evidence-based clinical guidelines. Cancer 104: 1129-1137, 2005

3. Benedet JL, Odicino F, Maisonneuve P, Beller U, Creasman WT, Heintz AP, Ngan HY and Pecorelli S: Carcinoma of the cérvix uteri. Int J Gynaecol Obstet 83 (Suppl 1): S41-S78, 2003.

4. Zhang B, Chen J, Ren Z, Chen Y, Li J, Miao X, Song Y, Zhao T, Li Y, Shi Y, et al: A specific miRNA signature promotes radioresistance of human cervical cancer cells. Cancer Cell Int 13: 118, 2013.

5. Yang CX, Zhang SM, Li J, Yang B, Ouyang W, Mei ZJ, Chen J, Dai J, Ke S, Zhou FX, et al: MicroRNA-320 regulates the radiosensitivity of cervical cancer cells C33AR by targeting $\beta$-catenin. Oncol Lett 12: 4983-4990, 2016

6. Pedroza-Torres A, Fernández-Retana J, Peralta-Zaragoza $\mathrm{O}$, Jacobo-Herrera N, Cantú de Leon D, Cerna-Cortés JF, Lopez-Camarillo C and Pérez-Plasencia C: A microRNA expression signature for clinical response in locally advanced cervical cancer. Gynecol Oncol 142: 557-565, 2016. 
7. Bousquet M, Harris MH, Zhou B and Lodish HF: MicroRNA miR-125b causes leukemia. Proc Natl Acad Sci USA 107: 21558-21563, 2010.

8. Hulanicka M, Garncarz M, Parzeniecka-Jaworska M and Jank M Plasma miRNAs as potential biomarkers of chronic degenerative valvular disease in Dachshunds. BMC Vet Res 10: 205, 2014.

9. Cowden Dahl KD, Dahl R, Kruichak JN and Hudson LG: The epidermal growth factor receptor responsive miR-125a represses mesenchymal morphology in ovarian cancer cells. Neoplasia 11: 1208-1215, 2009.

10. Betel D, Wilson M, Gabow A, Marks DS and Sander C: The microRNA.org resource: Targets and expression. Nucleic Acids Res 36: D149-D153, 2008.

11. Asgharian A, Banan M and Najmabadi H: Optimizing a lipocomplex-based gene transfer method into HeLa cell line. Cell J 15: 372-377, 2014.

12. Li G, Qiu Y, Su Z, Ren S, Liu C, Tian Y and Liu Y: Genome-wide analyses of radioresistance-associated miRNA expression profile in nasopharyngeal carcinoma using next generation deep sequencing. PLoS One 8: e84486, 2013.

13. Guo W, Xie L, Zhao L and Zhao Y: mRNA and microRNA expression profiles of radioresistant NCI-H520 non-small cell lung cancer cells. Mol Med Rep 12: 1857-1867, 2015.

14. McDermott N, Meunier A, Wong S, Buchete V and Marignol L: Profiling of a panel of radioresistant prostate cancer cells identifiesde regulation of key miRNAs. Clin Transl Radiat Oncol 2: 63-68, 2017

15. Maier P, Hartmann L, Wenz F and Herskind C: Cellular pathways in response to ionizin gradiation and their target ability for tumor radio sensitization. Int J Mol Sci 17: 102, 2016.

16. Ishigami T, Uzawa K, Higo M, Nomura H, Saito K, Kato $Y$, Nakashima D, Shiiba M, Bukawa H, Yokoe H, et al: Genes and molecular pathways related to radioresistance of oral squamous cell carcinoma cells. Int J Cancer 120: 2262-2270, 2007.
17. Skvortsova I, Skvortsov S, Stasyk T, Raju U, Popper BA, Schiestl B, Guggenberg Ev, Neher A, Bonn GK, Huber LA and Lukas $P$ : Intracellular signaling pathways regulating radioresistance of human prostate carcinoma cells. Proteomics 8: 4521-4533, 2008.

18. Moskwa P, Zinn PO, Choi YE, Shukla SA, Fendler W, Chen CC, Lu J, Golub TR, Hjelmeland A and Chowdhury D: A functional screen identifies miRs that induce radioresistance in glioblastomas. Mol Cancer Res 12: 1767-1778, 2014.

19. Shiiba M, Shinozuka K, Saito K, Fushimi K, Kasamatsu A, Ogawara K, Uzawa K, Ito H, Takiguchi $\mathrm{Y}$ and Tanzawa $\mathrm{H}$ : MicroRNA-125b regulates proliferation and radioresistance of oral squamous cell carcinoma. Br J Cancer 108: 1817-1821, 2013.

20. Fan Z, Cui H, Xu X, Lin Z, Zhang X, Kang L, Han B, Meng J, Yan Z, Yan X and Jiao S: MiR-125a suppresses tumor growth, invasion and metastasis in cervical cancerbytargeting STAT3. Oncotarget 6: 25266-25280, 2015.

21. Fan Z, Cui H, Yu H, Ji Q, Kang L, Han B, Wang J, Dong Q, Li Y, Yan Z, et al: MiR-125a promotes paclitaxel sensitivity in cervical cancer through altering STAT3 expression. Oncogenesis 5: e197, 2016.

22. Kraus A, Gross MW, Knuechel R, Münkel K, Neff F and Schlegel J: Aberrant p21 regulation in radioresistant primary glioblastoma multiforme cells bearing wild-type p53. J Neurosurg 93: 863-872, 2000.

23. Kokunai T, Urui S, Tomita $\mathrm{H}$ and Tamaki N: Overcoming of radioresistance in human gliomas by $\mathrm{p} 21 \mathrm{WAF} 1 / \mathrm{CIP} 1$ antisense oligonucleotide. J Neurooncol 51: 111-119, 2001. 